The Astrophysical Journal, 670:1420-1433, 2007 December 1

(C) 2007. The American Astronomical Society. All rights reserved. Printed in U.S.A.

\title{
MAGNETIC CYCLES IN THE SUN: MODELING THE CHANGES IN RADIUS, LUMINOSITY, AND $p$-MODE FREQUENCIES
}

\author{
D. J. Mullan, ${ }^{1}$ J. MacDonald, ${ }^{2}$ and R. H. D. Townsend ${ }^{1}$ \\ Received 2006 January 26; accepted 2007 August 16
}

\begin{abstract}
We report on the results obtained with a stellar evolution code in which cyclic magnetic fields are imposed in the convection zone of a $1.0 M_{\odot}$ star. Magnetic effects are incorporated in two ways: (1) the field pressure and energy density are included in the equations of hydrostatic equilibrium and conservation of energy; and (2) the field inhibits the onset of convection according to a prescription derived by Gough \& Tayler (1966). Inserting magnetic fields into the convection zone with strengths comparable to the observed global fields in the Sun, and assuming a simple depth dependence for the field strength, we find differences in luminosity and radius between nonmagnetic and magnetic models that are consistent in amplitude with the observed activity-related changes in the Sun. Using the same magnetic fields, and computing $p$-mode frequencies for nonmagnetic and magnetic models, we find that the frequencies in a magnetic model are larger than those for a nonmagnetic model. The frequency differences between nonmagnetic and magnetic models agree in sign, and overlap in magnitude and frequency dependence, with the shifts in frequency which have been observed in the Sun between solar minimum and solar maximum. We find that the luminosity variations are out of phase with the magnetic variations: in order to help reconcile this result with empirical solar data, we note that the global (poloidal) fields in the Sun are observed to pass through minimum values at times that correspond roughly with times of maximum toroidal fields.
\end{abstract}

Subject headings: stars: activity — Sun: magnetic fields

\section{INTRODUCTION}

Certain properties of the Sun are observed to vary during the course of a sunspot cycle. During the years 1978-2006, according to the $S O H O$ VIRGO World Wide Web site, the daily averages of the solar irradiance had excursions between a minimum value of $1362 \mathrm{~W} \mathrm{~m}^{-2}$ and a maximum value of $1368 \mathrm{~W} \mathrm{~m}^{-2}$, i.e., $\Delta L / L \approx 0.004$. Some of the largest excursions can be identified with the occurrence of individual sunspots on the disk. When yearly averages of $\Delta L / L$ are taken, the peak-to-peak amplitudes are found to be about 0.001 . Thus, depending on how averages are performed, the Sun exhibits fractional luminosity variations that, as regards amplitude, lie in the range 0.001-0.004 during the solar cycle. As regards the phase of the luminosity variations, the solar irradiance is observed to be largest when the sunspot count is at a maximum. Between solar minimum and solar maximum, the solar radius changes by less than 1 part in $10^{5}$ (Kuhn et al. 2004), and the photospheric temperature varies by $1.5-2 \mathrm{~K}$ (Gray \& Livingston 1997). The frequencies of $p$-modes are observed to increase at solar maximum compared to solar minimum: the increases are larger at higher frequencies, where the fractional increases are of order 1 part in $10^{4}$ (e.g., Dziembowski \& Goode 2005).

Since there is already clear evidence that magnetic fields can interfere significantly with convection at least in localized features such as sunspots (e.g., Mullan 1974), it seems plausible to associate the cyclic changes that are observed to occur in global solar properties also with magnetic field effects. In this regard, analyses of the activity-related changes in $p$-mode frequencies have led to some remarkably specific conclusions concerning the solar magnetic fields. For example, Li et al. (2003) conclude that "only a model that includes a magnetically modulated turbulent mech-

\footnotetext{
1 Bartol Research Institute, University of Delaware, Newark DE 19716

2 Department of Physics and Astronomy, University of Delaware, Newark DE 19716.
}

anism can agree with all the current observational data." Moreover, Dziembowski \& Goode (2005) conclude that the observed increases in mode frequencies require that at solar maximum, the field at a depth of about $5 \mathrm{Mm}$ below the photosphere must have a strength of 500-700 G.

The question is, can the effects of magnetic fields on stellar structure be modeled with enough reliability to account quantitatively for the changes which the Sun manifests in the course of a cycle?

Magnetic fields may affect the internal structure of a star in a variety of ways. For example, the radial gradient of magnetic pressure may contribute to hydrostatic support, or the fields may interfere with convective flows in such a way as to alter the efficiency of convection or the turbulent pressure. Various investigators have adopted different approaches to modeling magnetic effects in stars. In the first subsection below, we summarize some of these works, before going on to describing the approach we take here.

\subsection{Previous Modeling of Magnetic Effects in the Sun}

Here we summarize the work that has been done by a number of previous authors to include magnetic effects on the internal structure of the Sun.

\subsubsection{NASA Workshop (1980)}

At a NASA workshop dedicated to "Variations of the Solar Constant" in 1980 (Sofia 1981), various workers reported on numerical models in which inclusion of magnetic perturbations led to variability in the luminosity and radius of the Sun. Four overall categories can be identified as to how magnetic effects were incorporated in models. In one category, magnetic perturbations were included by reducing the efficiency of convection: this was modeled by reducing the mixing-length parameter $\alpha=l_{m} / H_{p}$, where $l_{m}$ is the mixing length, and $H_{p}$ is the local pressure scale height. A second category of magnetic models introduced tangled magnetic fields that are in equipartition with 
the kinetic energy of convection. A third category replaced the temperature gradient near the base of the convection zone with the radiative gradient. A fourth category considered perturbations in the solar core. In each case, results were reported for the accompanying changes in luminosity $\Delta L$ and in radius $\Delta R$. The numerical value of the ratio $W=\Delta \ln R / \Delta \ln L$ was considered to be of particular interest.

In a summary of published work, Gough raised the question, can observational data on luminosity and radius be used to pinpoint the "seat of the solar cycle"? Gough pointed out that, in the category of models where magnetic effects are included by lowering the mixing-length parameter $\alpha$, there is general agreement that reductions in $\alpha$ results in reductions in solar luminosity and radius (i.e., $\Delta L<0, \Delta R<0$ ). However, different magnetic models yielded quite different values of $W$, with numerical values differing by more than 100 , from $W \leq 5 \times 10^{-4}$ to $W=0.075$. In the second category of models, where the magnetic perturbations were modeled in terms of equipartition with convection, the conclusions were found to be quite different: in this case, magnetic fields led to positive values of $\Delta L$ and $\Delta R$. In the third and fourth categories, much larger values were obtained for the ratio $W$, viz., $0.20-0.53$. These are several orders of magnitude larger than in some models with altered $\alpha$.

Thus, depending on how and where one chooses to incorporate magnetic effects, the numerical value of $W$ may be quite different. As a result, Gough opined that "imminent observations" of $\Delta L$ and $\Delta R$ "will enable us to decide at least whether part of the dynamo process operates deep in the Sun."

\subsubsection{Dappen's Sensitivity Analysis (1983)}

A unified approach to modeling various classes of perturbations that might arise from magnetic fields in the Sun has been given by Dappen (1983). This work involves a Green's function analysis of how the four equations of stellar structure respond to a variety of disturbances. By linearizing these equations about an equilibrium solar model, and then adding a source term to represent a perturbation situated at a certain depth inside the Sun, Dappen shows that inversion of a matrix equation leads to solutions for $\Delta L, \Delta R$, and $W$ for any given perturbation. Each perturbation is assumed to have a sinusoidal dependence on time, with frequencies ranging over some 8 orders of magnitude: the corresponding periods range from 1 month to $10^{7} \mathrm{yr}$. Five classes of perturbations are considered: the first four involve localized disturbances in (1) pressure, (2) radius, (3) temperature, and (4) luminosity at a specific depth. The fifth class of perturbation involves (5) an alteration in the mixing-length parameter $\alpha$ uniformly throughout a shell that lies between the photosphere and a prescribed depth.

In terms of the frequency dependence, the sensitivity of the luminosity to various perturbations located at a specific depth depends on how the period of the perturbation compares to the convective envelope cooling time $t_{c}\left(=2 \times 10^{5} \mathrm{yr}\right)$. Perturbations in all five classes with periods less than $t_{c}$ lead to $\Delta L$ values that are independent of the period. But for longer periods, $\Delta L$ falls to zero in classes $1-3$, and 5, while for class $4, \Delta L$ rises to a constant value for long periods. The difference in behaviors has to do with how the structure of the Sun adjusts itself to the various perturbations.

Dappen finds that the amplitude of $\Delta L$ depends on how deeply below the surface the perturbation is applied.

As far as the sensitivity of radius to perturbations is concerned, a strong frequency dependence also emerges, but with significant differences from the behavior of $\Delta L$. For all five classes of perturbation, values of $\Delta R$ are independent of frequency at the longest periods. As the period of the perturbation shortens, $\Delta R$ decreases by $3-4$ orders of magnitude for classes 1 and 3-5.

In view of these differing sensitivities of $\Delta L$ and $\Delta R$, the ratio $W$ turns out to be very sensitive to frequency, increasing by 4-5 orders of magnitude as the period of the perturbations increases from 1 month to $10^{7} \mathrm{yr}$.

Moreover, Dappen finds that, by varying the depth beneath the surface at which the perturbation is applied, the value of $W$ can be made to vary by a few orders of magnitude.

Dappen's results provide a useful framework that helps one to understand modeling of the various types of magnetic perturbations reported by Gough (in Sofia 1981), as well as subsequent work by Endal et al. (1985), Lydon \& Sofia (1995), and Li \& Sofia (2001): all of these authors introduced magnetically related perturbations confined within narrow depth ranges. Dappen's perturbation approach was also used by Balmforth et al. (1996) to determine the changes in solar structure in response to certain internal thermal disturbances: going beyond Dappen, Balmforth et al. went on to determine how the structural changes would lead to shifts in $p$-mode frequencies. To calculate how magnetic fields lead to $p$-mode shifts, Balmforth et al. used variational methods developed by Gough (1990) and by Goldreich et al. (1991).

\subsubsection{Li et al. (2003)}

In an approach that incorporates a variety of progressively more detailed magnetic effects in a systematic manner, Li et al. (2003) compute solar models in which magnetic effects are incorporated by reducing turbulent velocities from a hydrodynamical model by a factor that depends on the level of magnetic activity. The residual turbulence is parameterized by a two-parameter $\left(f_{1}\right.$, $f_{3}$ ) function of depth. The residual turbulence is assumed to generate its own small-scale fields with an efficiency $f_{2}$, which is also a free parameter. The interaction between turbulence and radiative losses from a convective element is modeled in terms of a fourth parameter $f$. With various choices of the four free parameters, 18 distinct solar cycle models are computed and compared with observations. Two of the models are found to be consistent with observations: both of these models require specific (and nonzero) values for the four parameters.

\subsubsection{Virial Considerations}

The models described in $\S \S 1.1 .1-1.1 .3$ involved detailed modeling of the radial profile of various physical parameters. At a more global level, applications of the virial theorem to solar magnetism have yielded some general results that are of interest to the present paper.

The virial theorem states that for a star in equilibrium, the sum of gravitational potential energy $\Omega$, magnetic energy $E_{M}$, and (twice) the total of all types of thermal energy $K$ (including gas kinetic energy, turbulence, convective flows, rotation, and pulsation) is equal to zero. The question that is relevant here is, when the magnetic energy changes with time (as it does during the solar cycle), how are the changes in $E_{M}$ compensated by changes in $\Omega$ and $K$ ?

Steiner \& Ferriz-Mas (2005, hereafter SFM) quantify the changes in magnetic energy and radiant output from the Sun in the course of a cycle: it is interesting that these two changes are found to be comparable (they do not differ by more than an order of magnitude). This suggests that the structural and thermal changes caused by magnetic fields in the Sun are (almost) compensated by changes in the luminosity. SFM suggest a thermodynamic cycle whereby a deep-seated dynamo might help to explain how changes in magnetic energy might be converted into changes in solar radiative output in the course of a solar cycle. In 
a discussion of phase shifts, SFM suggest that the virial terms $E_{M}$ and $K$ may differ in phase by finite amounts, provided that $\Omega$ changes correspondingly. In support of the possibility of finite phase shifts, SFM cite a dynamo model by Brandenburg et al. (1992) in which the surface luminosity was found to lag behind $E_{M}$ by $43^{\circ}$ in phase.

Stothers (2006) shows that in the presence of magnetic variability on $11 \mathrm{yr}$ periods, the solar radius is smallest when the magnetic energy is maximum. However, when the variability occurs on long timescales (exceeding the envelope cooling time), the solar radius is greatest when the magnetic energy is maximum. We will refer to this result below $(\S 3.1)$ when we evaluate our model results.

\subsection{Aim of This Paper}

Our goal is to report on evolutionary calculations in which a star of $1 M_{\odot}$ is subjected to periodic magnetic fields in the convective envelope. In $\S 2$ we summarize the modeling approach that we adopt. This approach overlaps in some ways with the various papers reported above $(\S 1.1)$, but our work differs from those papers in the method we use to treat the magnetic interference with convection. In $\S 3$ we report on the changes in luminosity and radius that we find in our magnetic models. In $\S 4$ we compare our results with the empirical limits. In order to check that the models that we compute are reliable representations of the interior structure of the Sun, we report on an application of a pulsation code to our models in $\S 5$. Our principal aim in using the pulsation code is to undertake a differential study of the frequency shifts that arise between nonmagnetic solar models and magnetic solar models. We compare the results of our differential study with SOHO MDI data that indicate shifts in frequency between solar minimum and solar maximum. In $\S 6$ we discuss phase shifts and energy considerations. Our conclusions are presented in $\S 7$.

\section{EVOLUTIONARY MODELING OF THE INTERACTION BETWEEN MAGNETIC FIELDS AND CONVECTION}

Here we summarize the approach we take to incorporating magnetic field effects into an evolution code in order to determine how certain global parameters of the Sun are altered by magnetism. The code we use has been developed by one of us (J. M.) for general studies of stellar evolution. The magnetic version of the code was used in our earlier study of static magnetic fields in stars along the lower main sequence (Mullan \& MacDonald 2001, hereafter MM01).

The code uses OPAL opacities (Iglesias \& Rogers 1996) for temperatures above $7000 \mathrm{~K}$ and opacities prepared by D. R. Alexander at lower temperatures. The OPAL tables are interpolated using the routine written by Arnold Boothroyd, available from the OPAL Web site. Pressure ionization and electrostatic terms are included in the equation of state. Convection is treated by means of the mixing-length theory as formulated by Mihalas (1978): our choice of mixing-length parameter $\alpha$ is determined by requiring that the model reproduce the solar radius, luminosity, and age. In the code, once a value of $\alpha$ is chosen, the numerical value of $\alpha$ remains constant at all depths. In one set of models (M07), gravitational settling and element diffusion, including thermal diffusion, are incorporated for all elements using the formulation of Burgers (1969). In another set of models (M06), effects of settling and diffusion were excluded from the code. Resistance coefficients for the static screened Coulomb potential are from Paquette et al. (1986) for repulsive interactions and MacDonald (1991) for attractive interactions. Further details of the nonmagnetic code can be found in Lawlor \& MacDonald (2006).
In order to incorporate magnetic effects in the code, we add magnetic pressure and energy density terms to the equations of hydrostatic equilibrium and conservation of energy. In addition, the criterion for the onset of convection is modified to include magnetic effects according to a prescription derived by Gough $\&$ Tayler (1966). We now turn to a detailed description of that criterion.

\subsection{The Gough-Tayler Criterion}

As regards the onset of convection, we note that, in the absence of magnetic fields, the criterion for convective instability is given by the well-known Schwarzschild formula: $\nabla_{\text {rad }}>\nabla_{\text {ad }}$, where $\nabla=d \log T / d \log P$. The subscript "rad" denotes the temperature gradient which would be necessary in any locale in order to transport the local energy flux by radiative transport. The subscript "ad" denotes the adiabatic gradient.

In the presence of magnetic fields, it is energetically more difficult for convection to set in. A quantitative expression of this was derived by Gough \& Tayler (1966): using energy arguments, they showed that convective stability is ensured as long as $\nabla_{\text {rad }}$ does not exceed $\nabla_{\text {ad }}+\delta$, where $\delta$ is a positive definite quantity. Here the magnetic inhibition parameter $\delta$ is defined, in the presence of a vertical magnetic field $B_{v}$, by the ratio of magnetic to total energy density:

$$
\delta=\frac{B_{v}^{2}}{B_{v}^{2}+4 \pi \gamma P_{\mathrm{gas}}} .
$$

In this equation, $\gamma$ is the local ratio of specific heats.

In view of equation (1), increasing the vertical field strength makes $\delta$ larger. Therefore, as the vertical magnetic field increases in strength, $\nabla_{\text {rad }}$ must exceed $\nabla_{\text {ad }}$ by an increasingly large amount before convection sets in. Thus, equation (1) embodies the physical fact that magnetic fields make it more difficult for convection to occur. For this reason, we refer to $\delta$ as a "magnetic inhibition parameter." In the presence of nonzero $\delta$, convective efficiency is affected, and this leads to changes in stellar structure.

Our use of the Gough-Tayler (GT) criterion has the effect that our approach differs from that of Dappen (1983) in three distinct ways. First, Dappen retained the usual Schwarzschild criterion for the onset of convection, whereas we use the GT revision of the criterion. Second, Dappen reduced the value of $\alpha$, whereas we hold $\alpha$ fixed. Third, Dappen restricted the magnetic interaction with convection (as modeled by a change in $\alpha$ ) to layers between a certain depth and the surface, i.e., the changes in $\alpha$ were permitted only in relatively shallow layers of the Sun. Here, as we describe below, we pick a value of $\delta$ of order $10^{-5}$ and apply it essentially unchanged throughout the convection zone. By changing the Schwarzschild criterion in the way GT suggest, effects will be larger in our models in the deep convection zone. To see why this is so, we note that near the surface, where the difference $\Delta \nabla=\nabla_{\mathrm{rad}}-\nabla_{\mathrm{ad}}$ is much larger than $10^{-5}$, the use of the GT revision of the Schwarzschild criterion makes little difference to the local structure. However, in the deeper convection zone, where $\Delta \nabla$ becomes a small difference between two relatively large numbers, the introduction of a "correction" of order $10^{-5}$ in the Schwarzschild criterion is by no means a small effect. In essence, in any model with a given $\delta$, the changes introduced by the GT criterion amount to zerothorder corrections when we are considering layers in the Sun where $\nabla_{\text {rad }}-\nabla_{\text {ad }}$ in the nonmagnetic model has a magnitude of order $\delta$ or smaller. As a result, the structural changes in our models are more significant in the deeper layers of the convection zone. 
It is the threshold nature of the GT convective criterion that gives rise to the most significant structural changes between magnetic and nonmagnetic models. Consequences of including magnetic pressure are less significant: in cases where $\delta$ is of order $10^{-5}$, pressure changes contribute only at the level of 1 part in $10^{5}$.

\subsection{Time-Variable Magnetic Fields}

In the work of MM01, the possibility that magnetic fields might create detectable changes in stellar models was tested only in the case of fields which remain constant with time. The structural changes reported by MM01 referred to snapshots of magnetic stars after they had evolved for several billion years.

In the present paper, we extend the MM01 work in one sense, but restrict it in another sense. First, we depart from the static fields of MM01, and extend the discussion to time-dependent (specifically, periodic) fields. Second, we do not study stars with a range of masses: rather, we restrict attention here to stars of a single mass, $1 M_{\odot}$. Our goal is to explore the question, are there detectable cyclic effects in any of the physical parameters when magnetic fields are incorporated in a solar model?

To the extent that our modeling of magnetic effects has some overlap with certain aspects of Dappen's (1983) work, the frequencydependent results of Dappen (1983) provide a valuable reference check on our work. For example, our numerical results should replicate Dappen's conclusion that, in the presence of short-period variations, luminosity variations exhibit quite different frequency dependence from the variations in radius.

\subsection{Which Magnetic Fields Are Relevant to Global Structure?}

The empirical properties of the time variability associated with magnetic fields in the Sun are well known. The numbers of sunspots and flares cycle up and down on a period of 10-11 yr: these "magnetic activity" phenomena owe their origin to the existence of localized toroidal fields that grow as discrete flux tubes as a result of differential rotation in the course of the solar cycle. Averaged over an active region area, the strength of the toroidal fields is measured in hundreds of Gauss. The toroidal fields reverse sign, as a result of a complicated process (involving the decay of active regions) every $10-11$ yr.

Considerably weaker than the toroidal fields, the poloidal (global) fields also reverse sign on a 10-11 yr cycle.

It is important to note that in equation (1), the field that enters into the GT criterion is the vertical component. Horizontal components of the field do not interfere with convective onset, although they may change the cell topology (rolls vs. hexagons). Toroidal fields in the Sun are mainly horizontal and are, moreover, confined to discrete flux tubes, which erupt from time to time at the solar surface. On the other hand, the poloidal fields are global in nature, permeating all parts of the convection zone. The poloidal fields, which are certainly vertical in the polar caps, also retain significant vertical components throughout most of the volume of the convection zone. Only in the vicinity of the equator is there an absence of vertical fields.

This line of reasoning leads us to the conclusion that, when we wish to evaluate the quantity $\delta$ in equation (1) at the surface of the Sun, $\delta_{\text {surf }}$, it will be physically more meaningful to make use of the strength of the poloidal field of the Sun. We shall return below to the choice of an appropriate numerical value for $\delta_{\text {surf }}$.

\subsection{Choice of Radial Profile of Field Strength}

In order to apply the GT criterion to the structure of a star, we need to know not merely the surface value of $\delta$, but also how it varies as a function of radial location inside the star.
Three possible sets of radial profiles were discussed by MM01 in their modeling of lower main sequence stars. In sets 1 and $2, \delta$ was taken to be independent of the radial coordinate, i.e., $\delta(r)=$ $\delta_{\text {surf }}$. In sets 1 and 2, the magnetic field strength increases inwards monotonically from the surface as $[p(r)]^{1 / 2}$. The difference between sets 1 and 2 had to do with the radial component of the magnetic pressure gradient: in set 1, this gradient was ignored, whereas in set 2 , hydrostatic equilibrium was modified to incorporate the magnetic pressure gradient. In set 3 , magnetic pressure was included in hydrostatic equilibrium, and also $\delta$ was allowed to vary radially. The following radial profile was chosen:

$$
\delta(r)=\delta_{\text {surf }}\left[\frac{m(r)}{M_{*}}\right]^{2 / 3}
$$

where $m(r)$ is the stellar mass enclosed within radial distance $r$. According to equation (2), $\delta$ is largest at the surface and zero at the center of the star. Although no rigorous justification was provided for the above radial profile, MM01 offered a plausibility argument.

As it turned out, the results obtained by MM01 for low-mass stars showed no substantial differences between sets 1,2, or 3: all three sets agreed in the sense that the larger the choice of $\delta_{\text {surf }}$, the more the luminosity and radius differed from the standard (nonmagnetic) model of the same mass. Thus, apart from sensitivity to $\delta_{\text {surf }}$, the effects of magnetic fields on stellar structure were found to be insensitive to the choice of the radial profile for $\delta(r)$. The fact that results from sets 1 and 2 were found to be similar indicates that incorporating magnetic pressure into hydrostatic equilibrium is not the dominant effect in our models: the dominant structural effect has to do with the inclusion of the GT revision of the Schwarzschild criterion.

In the present work there are two important differences from the work of MM01. First, in MM01, the focus was on stars in which the convective envelope occupies most (or all) of the stellar mass: here we focus on the Sun, where the convection zone includes only $1 \%-2 \%$ of the overall mass. Second, whereas the fields in MM01 were static, we deal here with time-variable fields: moreover, the timescales for variability are as short as $10 \mathrm{yr}$. Now, the radiative interior of the Sun has a magnetic diffusion time ( $\tau_{\text {mag }} \approx L^{2} / \eta$, where $\eta$ is the electrical resistivity), which is orders of magnitude longer than $10 \mathrm{yr}$. Therefore, the time-variable fields are mainly confined to the convection zone. We have performed a number of numerical experiments to test the effects of using different ways of cutting off the magnetic field in the radiative interior. Because the dominant effect of the magnetic field is inhibition of convection, results are insensitive to the details of the implementation of the cutoff in the radiative interior. We settled on multiplying $\delta(r)$ by a Gaussian cutoff factor,

$$
f_{\text {cut }}= \begin{cases}1, & m \geq m_{\text {cut }}, \\ \exp \left[-c\left(m_{c u t}-m\right)^{2}\right], & m<m_{\text {cut }} .\end{cases}
$$

Here $m_{\text {cut }}$ is the value of the mass variable at the base of the surface convection zone and $c$ is a parameter that controls how quickly magnetic effects go to zero. Also, $m$ and $m_{\text {cut }}$ are measured in units of stellar mass. The particular values we used in the present work are $c=10^{2}$ and $m_{\text {cut }}=0.9762 M_{\odot}$. With these values, the value of $\delta$ falls below the surface value by factors of 10 and 100 at the locations where the mass coordinate equals 0.83 and $0.77 M_{\odot}$.

In the convection zone, for the sake of continuity with MM01, we retain the radial profile of $\delta$ as given in equation (2). However, 
since $m(r) / M_{*}$ is confined to the range $0.98-1.0$ in the convection zone of the Sun, this implies that $\delta(r)$ is essentially constant, and equal to $\delta_{\text {surf }}$, throughout the convection zone. Following the rules of set 3 in MM01, we retain the gradient of magnetic pressure in the equation of hydrostatic equilibrium. Moreover, because we are dealing here with time-variable magnetic fields, it is important (see Dappen 1983) to modify the energy equation: with this in mind, our magnetic models include the magnetic energy density as well as the internal energy density, and the models include the magnetic pressure, as well as the thermal pressure in the energy conservation equation.

\subsection{Choice of Temporal Variability of Field Strength}

We choose the following sinusoidal behavior as a function of time:

$$
\delta(r, t)=\delta(r)[1-\cos (2 \pi t / \tau)]
$$

Note that at time $t=0$, the field is zero: the field increases sinusoidally to its maximum value at $t=0.5 \tau$, and then decreases sinusoidally to zero at $t=\tau$. Thus, minimum field strengths are present at times $t / \tau=0,1,2, \ldots$, while the strongest fields occur at times $t / \tau=0.5,1.5,2.5, \ldots$ The form of equation (4) means that in models where we assign a mean value of (say) $\delta_{\text {surf }}=0.01$, the value of $\delta$ in the convection zone varies between a maximum value of 0.02 and a minimum value of 0 during one cycle.

The periods that we select for the variation in magnetic field strength range from an upper limit of $10^{8} \mathrm{yr}$ to a lower limit of $10 \mathrm{yr}$.

\section{EVOLUTION OF THE SUN WITH CYCLIC MAGNETIC FIELDS: RESULTS FOR RADIUS AND LUMINOSITY}

Here we report on the changes in solar radius and luminosity that occur in our models during the course of the solar cycle.

Most of the results to be presented below refer to a solar model, which we refer to as the "M07 model." M07 was constructed by evolving a grid of pre-main-sequence star models, all with initial $Z=0.0200$, but different values of $\alpha$ and initial helium mass fraction, $Y_{0}$. In these calculations element diffusion (including thermal diffusion) and gravitational settling of all species were followed. The values of $\alpha$ and $Y_{0}$ that give the correct luminosity and radius for the Sun at age 4.6 Gyr were found by interpolation. The M07 model was then constructed by evolving a model with the interpolated parameters from the pre-main-sequence to the solar age. The surface abundances of the M07 solar model are $X=0.7218$, $Y=0.2598$, and $Z=0.0183$.

A second model, M06, was computed by evolving a $1 M_{\odot}$ star with $X=0.7050, Z=0.0200$, and $\alpha=1.5$ from the pre-mainsequence to the point at which its luminosity is $1 L_{\odot}$. In computing the M06 model, the effects of element diffusion and gravitational settling were excluded.

In order to reduce the effects of transient behavior in the models, two preliminary steps are taken before we turn on the cyclic fields. First, the solar model is allowed to reach thermal equilibrium. Second, a static field distribution is added with strength corresponding to the chosen value of $\delta_{\text {surf }}$. We refer to this equilibrium static model as a "precycle" model. Because of the presence of the nonzero field, the global properties of the precycle model differ slightly from those of the current Sun: as a result, the mean radii and luminosities in Figures 1, 2, and 4 differ slightly from $R_{\odot}$ and $L_{\odot}$. Once the precycle model is available, we then turn on a sinusoidal field, and follow it for 10 periods.

\subsection{Magnetic Cycles and Radius: Amplitude and Phase}

In Figure 1 we show how the (logarithm to base 10 of the) radius of the solar model changes as a function of time for a fixed value of $\delta_{\text {surf }}(=0.01)$ and for a range of cycle periods $\tau$ from $\tau=10^{7}-10 \mathrm{yr}$. Along the time axis, the abscissa for each model is the actual time divided by the cycle period in that model. Moving to a weaker field, $\delta_{\text {surf }}=0.001$, we obtain the results shown in Figure 2. As expected, in the presence of a weaker field, the amplitudes $\Delta R$ of the cyclic changes in radii are smaller.

In Figure 3 we show how the relative peak-to-peak amplitude $\Delta R / R$ depends on $\tau$ for a range of values of $\delta_{\text {surf }}$. For $\tau>10^{7} \mathrm{yr}$, the models indicate that $\Delta R / R$ is independent of $\tau$, and scales linearly with $\delta_{\text {surf. }}$ Our model results can be described by

$$
\Delta R / R \approx 20 \delta_{\text {surf }}
$$

For cycle periods between $\tau=10^{7}$ and $\tau=10^{6} \mathrm{yr}$, there is a transition to power-law dependence on $\tau$, although the linear dependence on $\delta_{\text {surf }}$ remains:

$$
\Delta R / R \approx 2 \times 10^{-4} \tau^{0.8} \delta_{\text {surf }},
$$

where $\tau$ is measured in yr. Below $\tau=10^{5} \mathrm{yr}$, there is a second transition in which $\Delta R / R$ becomes independent of $\tau$ again, such that

$$
\Delta R / R \approx 1.3 \delta_{\text {surf }}
$$

At a value of $\tau$ that depends on $\delta_{\text {surf }}$, there is a further transition to a power-law dependence on $\tau$.

Are these scalings from our numerical models consistent with Dappen's (1983) sensitivity analysis? We can hardly expect to find complete consistency because our use of the GT criterion cannot be accommodated precisely into any of the five classes of perturbation in Dappen's work. Nevertheless, inspection of Dappen's Figure 3 indicates that for all five classes of his perturbations, $\Delta R / R$ reaches its largest value, and becomes independent of frequency, for cycles with the longest periods, just as we find in Figure 3. For four classes of perturbation, Dappen finds that, at intermediate periods, $\Delta R / R$ increases with increasing $\tau$, roughly as a power law: this is consistent with equation (6) above. It appears that our model results exhibit frequency dependences that overlap with the range of behaviors reported by Dappen.

As regards the phase of the magnetically induced radius variations, from Figures 1 and 2, we see that the phase at which radius is maximum depends on cycle period. For long periods, $\tau>10^{7} \mathrm{yr}$, maximum radius occurs when the field is strongest, i.e., at times $t / \tau=0.5,1.5,2.5, \ldots$. However, as $\tau$ decreases from $\sim 10^{7} \mathrm{yr}$ to $\sim 10^{4} \mathrm{yr}$, maximum radius occurs later in the cycle and for $\tau<10^{4} \mathrm{yr}$, maximum radius occurs when the field is minimum, i.e., at times $t / \tau=0,1,2, \ldots$ In particular, for the case of a 10 yr cycle, our models predict that the solar radius should be smallest when the field is strongest.

Dappen (1983) does not provide enough information to determine if this phase behavior is consistent with his work. However, the phase shifts in radius that our models exhibit have a frequency dependence that is entirely consistent with the behavior reported by Stothers (2006) based on virial considerations.

\subsection{Magnetic Cycles and Luminosity: Amplitude and Phase}

In Figure 4 we show the (logarithm to base 10 of the) luminosity as a function of time when periodic magnetic fields are 

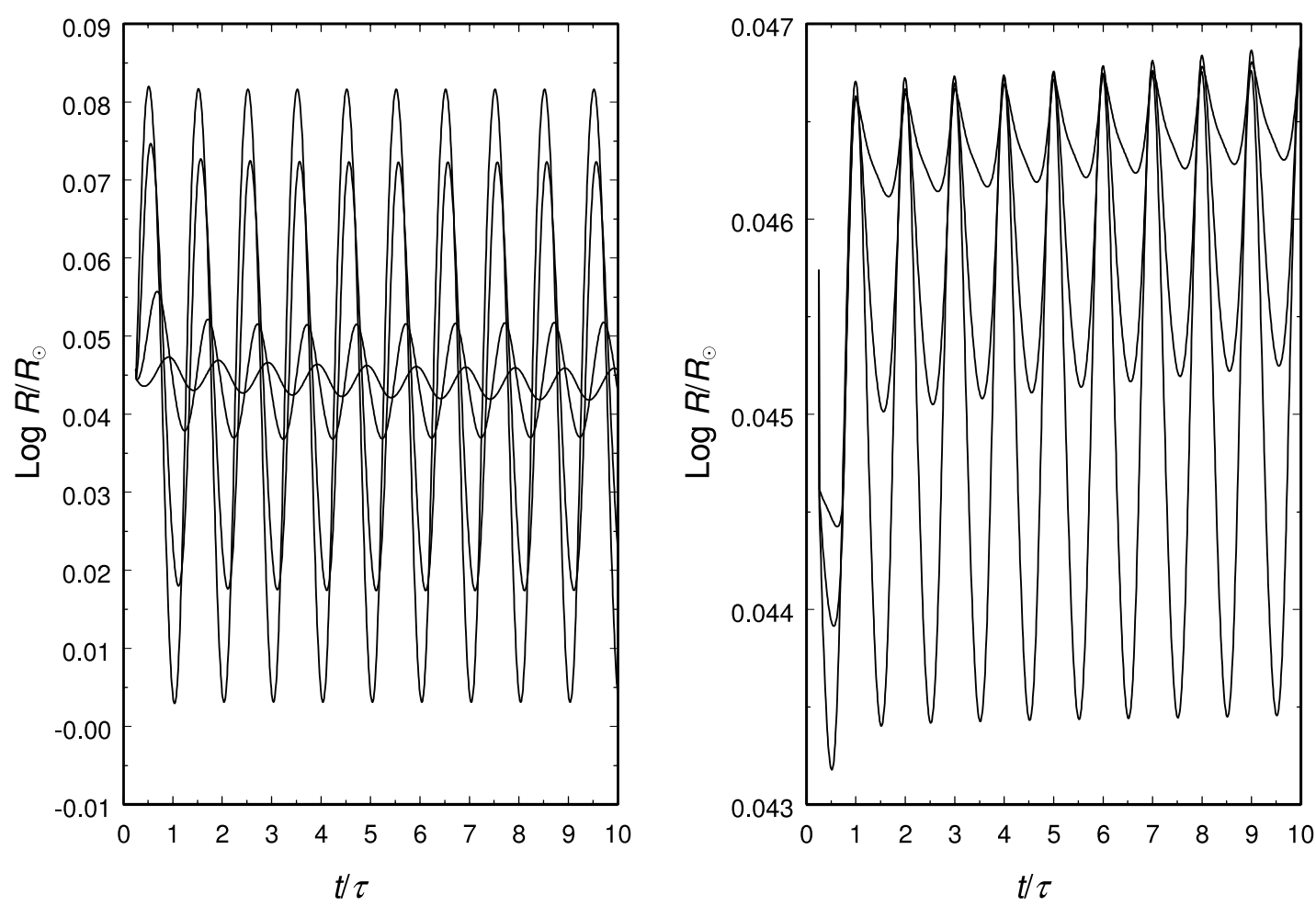

FIG. 1.- Temporal variations in the radius of a solar model with a periodic magnetic field. The mean surface value of the magnetic inhibition parameter $\delta$ (see eq. [1]) is 0.01 for all curves. The four curves in the left-hand panel in order of decreasing amplitude have periods $\tau=10^{7}, 10^{6}, 10^{5}$, and $10^{4}$ yr. The three curves in the right-hand panel in order of decreasing amplitude have periods $\tau=10^{3}, 10^{2}$, and $10 \mathrm{yr}$. Time is plotted in terms of the cycle period in each case.

introduced into our solar model. There are actually five distinct curves plotted in Figure 4 corresponding to periods of $10,10^{2}$, $10^{3}, 10^{4}$, and $10^{5} \mathrm{yr}$, but careful inspection is required to spot the differences. The abscissa is elapsed time in units of the period. As inspection of Figure 4 shows, the temporal changes in luminosity are essentially frequency-independent for periods in the range $10^{1}-10^{5} \mathrm{yr}$. This is entirely consistent with Dappen's conclusions (his Fig. 1) for periods shorter than $2 \times 10^{5} \mathrm{yr}$.

The five curves in Figure 4 were all computed for the case $\delta_{\text {surf }}=1.0 \times 10^{-5}$. We note that the peak-to-peak amplitude of the luminosity variation $\Delta \log _{10} L / L$ is found in all cases to be $3.5 \times 10^{-4}$. Converting to natural logarithms, this corresponds to $\Delta L / L=8.1 \times 10^{-4}$.

In Figure 5 we show how $\Delta L / L$ depends on $\tau$ for a range of values of $\delta$. We find that $\Delta L / L$ scales linearly with $\delta_{\text {surf }}$. For periods between 10 and $10^{5} \mathrm{yr}, \Delta L / L$ is independent of $\tau$, and increases linearly with $\delta_{\text {surf }}$. This leads us to fit our results with the formula

$$
\Delta L / L \approx 81 \delta_{\text {surf }}
$$

For longer periods, $\tau>10^{6} \mathrm{yr}$, we find that $\Delta L / L$ decreases with increasing $\tau$ : the decrease can be fitted by means of a power law in $\tau$,

$$
\Delta L / L \approx 6.0 \times 10^{5} \tau^{-0.63} \delta_{\text {surf }},
$$

where $\tau$ is measured in yr.

The frequency dependences in equations (8) and (9) are consistent with Dappen's results for four of his five classes of perturbation. We reiterate that the kind of magnetic perturbations we are introducing into the convection model do not overlap precisely with any of the perturbations that Dappen considers.
Therefore, we do not expect to find perfect correspondence between Dappen's conclusions and ours.

As regards the phase of the luminosity variations, we find that, for periods of $10^{1}-10^{5} \mathrm{yr}$, the luminosity is largest when the magnetic field is weakest, i.e., at times $t / \tau=1,2,3, \ldots$, in Figure 4 . The luminosity minima in Figure 4 correspond to time intervals when the global field in the star has maximum strength. Thus, magnetic fields and luminosity are $180^{\circ}$ out of phase with each other. For longer periods, our model results (not shown) indicate that the maximum luminosity occurs at a phase of the cycle slightly before the magnetic field minimum.

In comparison with Dappen's results for phases, his Figure 2 indicates that, depending on the class of perturbation, the changes in luminosity are in some cases in phase with the perturbation, but in other cases, they are $180^{\circ}$ out of phase. The latter occurs in cases where the perturbation is introduced into the hydrostatic equation: this is one of the effects we incorporate here.

\section{COMPARISON BETWEEN MODEL OUTPUTS AND SOLAR DATA}

In this section, we compare our results with radius and luminosity data that are available for the Sun.

\subsection{Numerical Value of $\delta_{\text {surf }}$ in the Sun}

For purposes of comparison with the Sun, we first need to estimate an appropriate numerical value of $\delta_{\text {surf }}$. As was remarked above, the appearance of vertical fields in the GT criterion suggests that it is appropriate to use the poloidal field of the Sun in evaluating $\delta_{\text {surf }}$. In situ interplanetary data indicate that the maximum polar cap field strength is in the range 6-12 $\mathrm{G}$ (Hundhausen, 1977). In view of this, it seems plausible to consider that the 

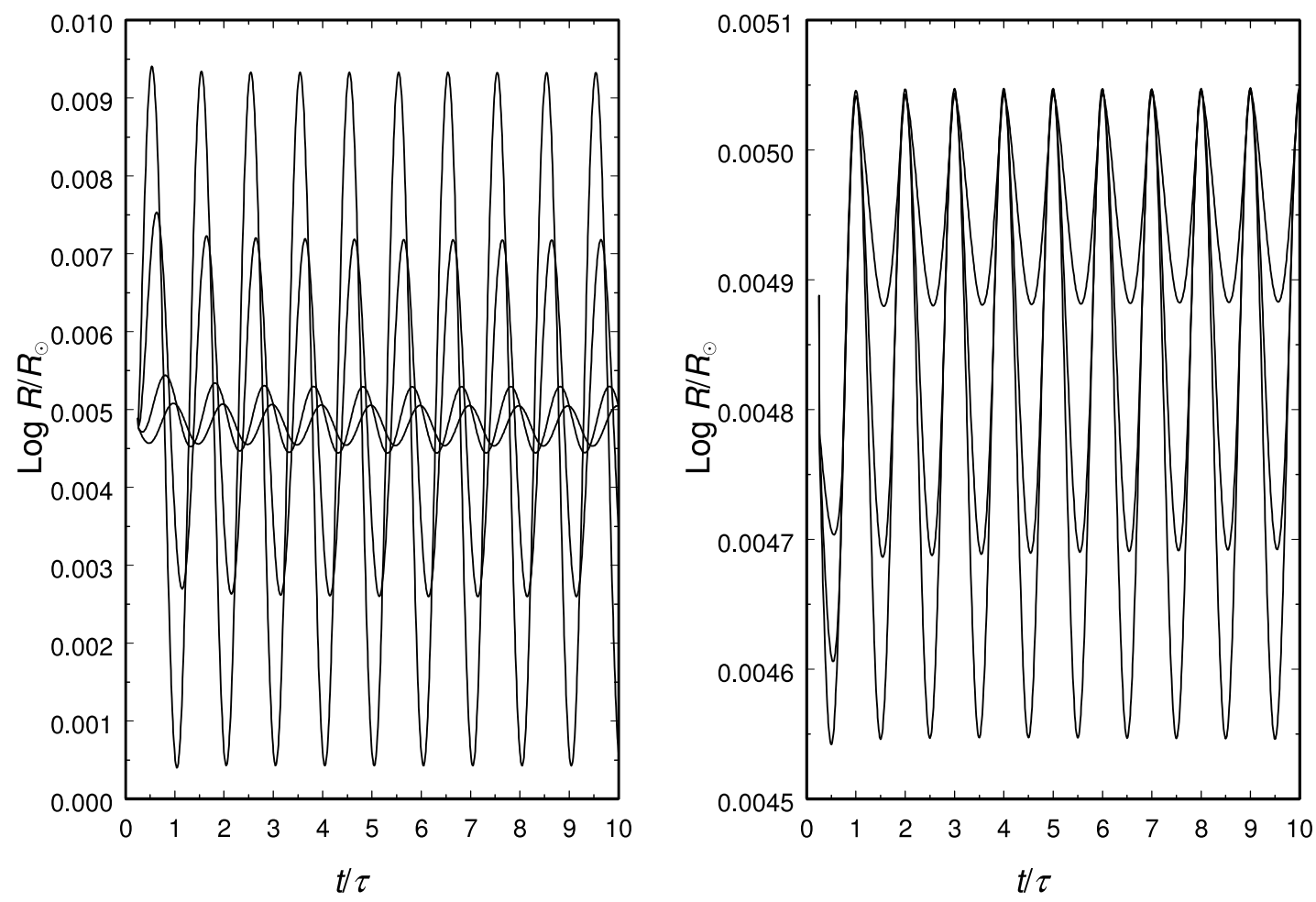

FIG. 2.- Temporal variations in the radius of a solar model with a periodic magnetic field. The mean surface value of the magnetic inhibition parameter $\delta$ (see eq. [1]) is

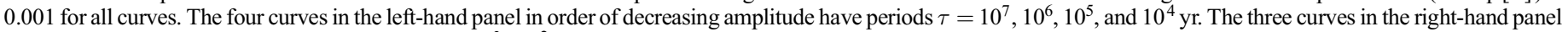
in order of decreasing amplitude have periods $\tau=10^{3}, 10^{2}$, and $10 \mathrm{yr}$. Time is plotted in terms of the cycle period in each case.

surface field that is relevant to us here varies, in the course of the solar cycle, between zero and 6-12 G.

Now, the photospheric pressure $P_{\text {gas }}$ is $1.2 \times 10^{5}$ dyn $\mathrm{cm}^{-2}$ (Foukal 1990). Combining the above value of maximum polar field strength and $P_{\text {gas }}$, we find that the maximum value of $\delta$ in the solar photosphere is $\delta_{\max } \approx(1-5) \times 10^{-5}$. In view of the notation we use in equation (4), where the maximum value of $\delta$ is $\delta_{\max }=2 \delta_{\text {surf }}$, we find that $\delta_{\text {surf }}$ has a numerical value of about $(0.5-2.5) \times 10^{-5}$. Also for the Sun, $\tau \approx 10 \mathrm{yr}$.

Are there ways to check that the above choice of the maximum $\delta_{\text {surf }}$ is in fact appropriate for the Sun? We offer two possibilities.

First, we refer to the work of Dziembowski \& Goode (2005). With a value of $\delta_{\max }=(1-5) \times 10^{-5}$ at the surface, and recalling that the radial profile in equation (2) leads to essentially invariant $\delta$ with depth, we see that the maximum value of $\delta$ at a depth of $5 \mathrm{Mm}$ below the photosphere is not measurably different from $(1-5) \times 10^{-5}$. At a depth of $5 \mathrm{Mm}$ in our solar model, we find that the local gas pressure is $2.8 \times 10^{8} \mathrm{dyn}^{-2}$. Combining this pressure with the maximum $\delta$ value, we find that the vertical field strength at a depth of $5 \mathrm{Mm}$ in our model at solar maximum is $B_{v}(5 \mathrm{Mm}) \approx\left(4 \pi \gamma \delta_{\max } p_{\text {gas }}\right)^{1 / 2} \approx 240-540 \mathrm{G}$, using $\gamma=5 / 3$. If the changes in magnetic field between solar minimum and solar maximum are isotropic (e.g., Dziembowski \& Goode 2005), then the total equivalent field strength corresponding to a given $B_{v}$, including horizontal components of comparable strength, would be of order $\sqrt{2} B_{v} \approx 340-760 \mathrm{G}$. This overlaps quite well with the range $500-700 \mathrm{G}$ at $5 \mathrm{Mm}$ derived by Dziembowski \& Goode (2005).

An independent check on an appropriate value of $\delta_{\max }$ in the Sun can be obtained by considering conditions at the base of the convection zone, where the solar dynamo is believed to originate. Although Coriolis forces tend to drive rising flux ropes toward the poles of the Sun (Choudhuri \& Gilman 1987), it is well known that active regions on the Sun's surface are confined to low latitudes. In order to replicate this empirical constraint, it is necessary to invoke strong buoyancy forces, which overcome the Coriolis tendencies. This requirement can be satisfied if the field strengths at the base of the convection zone (at radial location $r_{\mathrm{CZ}}$ ) are in excess of a limiting value: depending on thermal energy considerations,

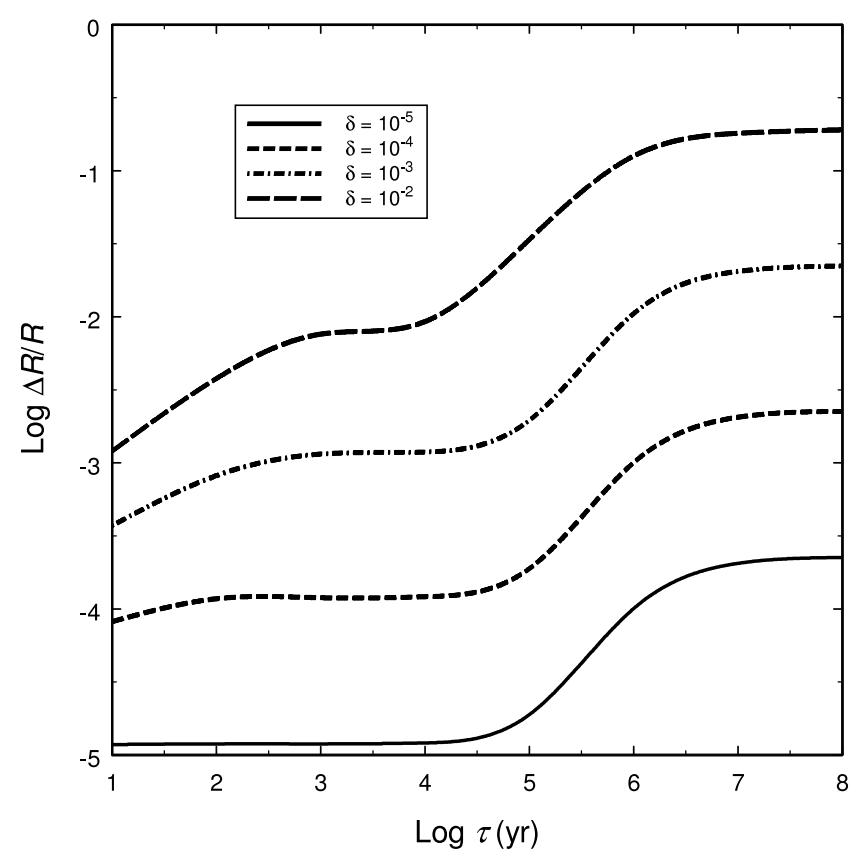

FIG. 3.-Amplitude of relative variations in radius $\Delta R / R$ of a solar model star with a periodic magnetic field plotted against cycle period $\tau$ for $\delta=10^{-5}, 10^{-4}$, $10^{-3}, 10^{-2}$. 


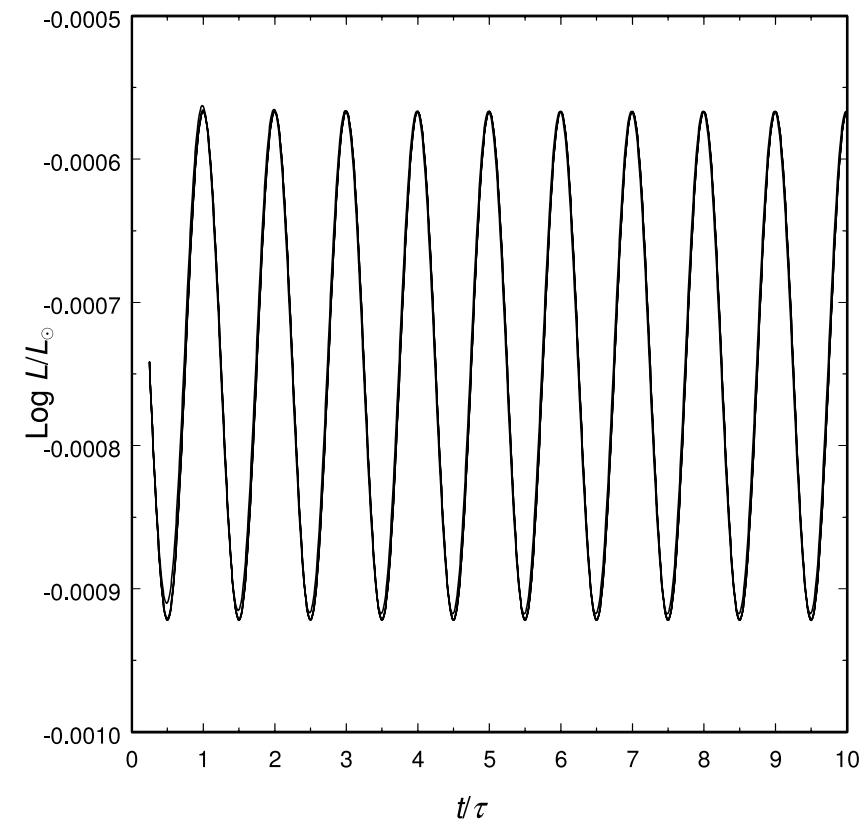

FIG. 4.- Temporal variations in the luminosity of a solar model with a periodic magnetic field. The mean surface value of the magnetic inhibition parameter $\delta$ (see eq. [1]) is $1.0 \times 10^{-5}$ for all curves. There are five curves plotted here, with periods $\tau=10,10^{2}, 10^{3}, 10^{4}$, and $10^{5}$, yr, but the differences between curves are small. Time is plotted in terms of the cycle period in each case.

Choudhuri \& Gilman find that fields of order $(1-2) \times 10^{5} \mathrm{G}$ could satisfy this condition. Work by Caligari et al. (1998) also indicates that the dynamo generates fields of order $(1-2) \times 10^{5} \mathrm{G}$ at the base of the convection zone. Now, the gas pressure at the base of the convection zone is $\approx 5 \times 10^{13} \mathrm{dyn}^{-2}$ (e.g., Bahcall et al. 2006). According to equation (1), a field of $(1-2) \times 10^{5} \mathrm{G}$ in the presence of such gas pressure corresponds to $\delta\left(r_{\mathrm{CZ}}\right)=(1-4) \times$ $10^{-5}$. Since our models assume essentially constant $\delta$ throughout the convection zone, it is legitimate to compare $\delta\left(r_{\mathrm{CZ}}\right)$ with the surface estimate of $\delta_{\max }=(1-5) \times 10^{-5}$. Such a comparison indicates satisfactory overlap between the two estimates.

The range $\delta_{\text {surf }}=(0.5-2.5) \times 10^{-5}$ is consistent with the above three independent estimates.

\subsection{Solar Cycle Changes in Solar Radius}

Now that we have a value for $\delta_{\text {surf }}$, we see from equation (7) that our models predict the peak-to-peak radius variations in the Sun during the solar cycle to be $\Delta R / R \approx(0.7-3) \times 10^{-5}$. Thus, the change in the solar angular radius (which has a mean value of about $1000^{\prime \prime}$ at $1 \mathrm{AU}$ ) between minimum and maximum activity is predicted to be 7-30 mas.

Kuhn et al. (2004) have reported, on the basis of data obtained in space, that during solar cycle 23 , between solar minimum and solar maximum, the radius of the Sun did not change by more than 7 mas. Other investigators have reported larger amplitudes, in some cases by more than an order of magnitude (see references in Stothers 2006), but the distorting effects of the Earth's atmosphere are difficult to correct for with sufficiently high confidence. The data of Kuhn et al. (2004) provide the most stringent test of our model predictions. Our model predictions are marginally consistent with the limit of Kuhn et al. (2004).

As regards the phase of the radius, we recall $(\S 3.1)$ that for an 11 yr magnetic cycle, the solar radius should be smallest when the magnetic field is strongest. Since Kuhn et al. (2004) report only an upper limit, we cannot use Kuhn et al.'s data to test this aspect of our work. Interestingly, Stothers (2006) notes that among

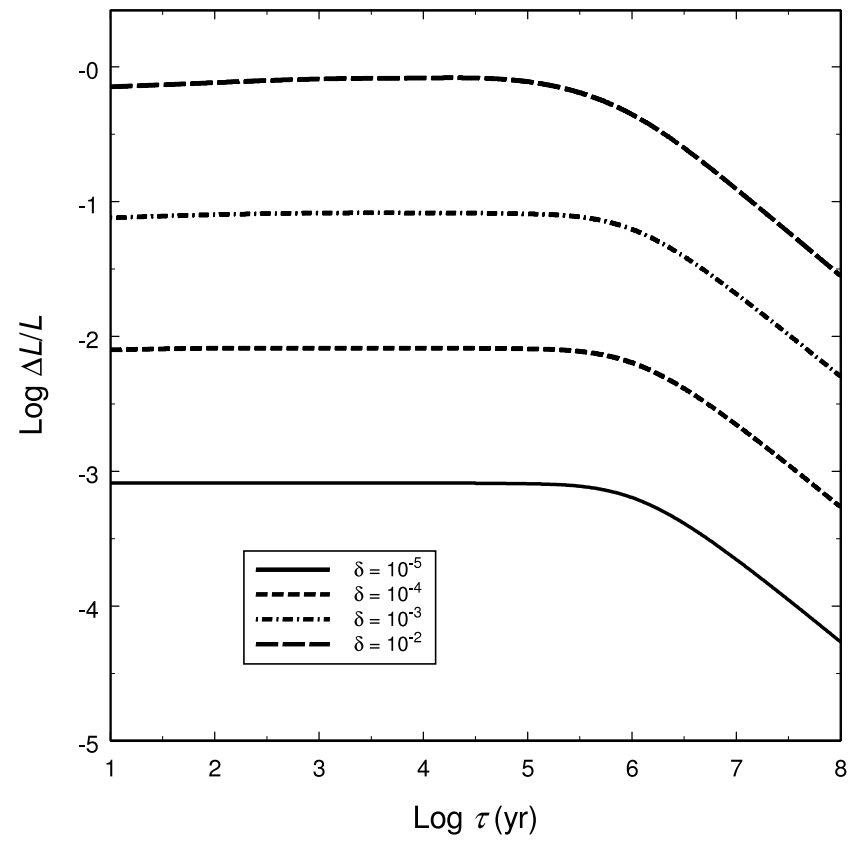

FIG. 5.-Amplitude of relative variations in luminosity $\Delta L / L$ of a solar model star with a periodic magnetic field plotted against cycle period $\tau$ for $\delta=10^{-5}$, $10^{-4}, 10^{-3}, 10^{-2}$.

those who have used nonspacecraft data, four groups of observers have claimed that the solar radius varies in phase with surface activity, seven groups of observers have reported radius changes in antiphase with surface activity, while four groups of observes have reported no significant change at all.

\subsection{Cyclic Changes in Solar Luminosity: Amplitudes}

Inserting $\delta_{\text {surf }}=(0.5-2.5) \times 10^{-5}$ in equation (8), our models predict that the peak-to-peak variations in solar luminosity are $\Delta L / L \approx(0.4-2) \times 10^{-3}$. Empirically, between 1978 and 2006 , the peak-to-peak changes in $\Delta L / L$ were observed to be $(1-4) \times$ $10^{-3}$. Thus, in quantitative terms, the predictions of our solar model as regards the amplitude of luminosity variations are consistent with the observed solar cycle variations.

If changes in radius and changes in effective temperature $T$ are in phase, then $\Delta L / L=2 \Delta R / R+4 \Delta T / T$ allows us to estimate the changes in $T$ that should occur in the course of a solar cycle. Inserting the maximum value of $\Delta R / R$ from our models we find that the term $2 \Delta R / R$ has at most a value of 0.00006 . Thus, $\Delta L / L \approx$ 0.002 is due mainly to changes in $T: \Delta T / T=0.002 / 4=0.0005$. In the presence of phase shifts between luminosity, radius, and temperature, the amplitude $\Delta T$ will be smaller than this. The corresponding value of $\Delta T$ is less than $3 \mathrm{~K}$, consistent with the temperature changes of 1.5-2 K reported by Gray \& Livingston (1997) for the photosphere.

\subsection{Cyclic Changes in Solar Luminosity: Phases}

According to our models, the phase of the luminosity variation is such that the Sun is predicted to be least luminous when the global magnetic field strength is strongest. At first sight, the solar data appear to contradict this prediction: the Sun is observed to be most luminous when sunspot counts are at their maximum. We recall that the sunspots are associated with the strongest fields on the surface of the Sun. Thus, there appears to be a phase discrepancy between our predictions of luminosity variations and the observations. However, we also recall that the sunspot fields are highly localized: the largest sunspot group ever recorded (in 1947) 
had a maximum area of 6132 millionths of the visible hemisphere (Bray \& Loughhead 1979), i.e., a maximum area of only 0.003 times the surface area of the Sun. More than $95 \%$ of all sunspots are 10 times smaller than this limit.

We return to this topic in $\S 6$.

\section{5. $p$-MODE FREQUENCIES}

An important goal of the present study is to quantify magnetically induced shifts in $p$-mode frequencies in a model of the present-day Sun.

Now that we have obtained models of the Sun, $p$-mode frequencies can be calculated by applying the latest version of the BOOJUM pulsation code developed by one of the authors (R. H. D.T.; see, e.g., Townsend \& MacDonald 2006). This code solves the linearized equations for nonadiabatic pulsation (see Unno et al. 1989) using a finite-difference approach. The code solves for the mode frequencies in terms of a unit $\nu_{d}=(1 / 2 \pi) \times$ $\left(\mathrm{GM}_{\odot} / R^{3} \odot\right)$, which is the inverse of a dynamical timescale. Following Ando \& Osaki (1975), radiative heat transport is treated using the Eddington approximation, which is valid in optically thick and optically thin layers. To model convective heat transport, BOOJUM adopts a frozen-convection approach where the perturbation to the convective source term in the energy equation is neglected. Any errors introduced by either of these approximate treatments of energy transport are likely to be small - indeed, we find the differences between calculated frequencies and the corresponding adiabatic frequencies (also calculated by BOOJUM as a configurable option) are, in the worst case, on the order 1 part in $10^{3}$. However, in the present work, we are primarily interested in a differential study of the frequency shifts between nonmagnetic and magnetic models. When such shifts are computed using adiabatic and nonadiabatic versions of BOOJUM, the differences in the magnitudes of the shifts are less than 1 part in $10^{5}$.

In order to apply the pulsation code to our magnetic models, a data file was prepared from each magnetic model to serve as input to the pulsation code. The input data file included the radial profile of (among other quantities) the total pressure, equal to the sum of gas pressure plus a magnetic pressure. (The correction for magnetic pressure relative to gas pressure is of order $\delta / 2 \gamma=$ $0.3 \delta$. Thus, for typical values of $\delta=2 \times 10^{-5}$ in the current paper, the correction for magnetic pressure is less than $10^{-5}$ times the gas pressure.) Moreover, thermodynamic derivates with respect to pressure, which are part of the input data file, also included the magnetic pressure. And in evaluating the radial profile of the Brunt-Vaisala frequency (also needed as input for the pulsation code), magnetic effects were explicitly included. As regards the actual pulsation code itself, apart from inputting the magnetically modified structural variables, we made no specific modification to BOOJUM. To be sure, a magnetic field will have some influence on the pulsation dynamics, through the appearance of additional forces associated with magnetic pressure and tension. Anisotropies associated with magnetic fields influence magnetoacoustic modes in complicated ways depending on whether the fields are horizontal, or vertical, or force-free, or statistically isotropic (see the Appendix of Goldreich et al. 1991). As $p$-modes propagate through the Sun between the inner and outer edges of an acoustic cavity, the waves will encounter a variety of directions relative to the local magnetic field, sometimes propagating parallel to the local field, sometimes perpendicular. In such conditions, full inclusion of the perturbed Lorentz forces into the pulsation code would be a very complicated undertaking. We have not included these perturbed Lorentz forces in our pulsation modeling.

As justification for neglecting the perturbed Lorentz forces in the present work, we note that the relative changes in frequency associated with magnetic effects are determined by the squared ratio of Alfvén speed to sound speed (see eq. [8] of Goldreich et al. 1991). This squared ratio is identical with our parameter $\delta$, as defined in equation (1). Now, for the typical weakly magnetic cases we discuss here, the values of $\delta$ are of order $10^{-5}$. As a result, the dynamical effects of a magnetic field in the pulsation code would result in changes to the calculated pulsation frequencies at the $10^{-5}$ level. The observed frequency shifts between solar minimum and solar maximum have relative magnitudes which are an order of magnitude larger than this.

In the present paper, the magnetic field influences the pulsation frequencies indirectly, via changes in the equilibrium structure due to application of the GT criterion. As discussed in $\S 2.1$, structural changes introduced by this criterion can be significant, even for the small $\delta$ values we consider. Since these changes alter the structure of the Sun in a nonhomologous manner ( $\operatorname{see} \S 5.3$ below), and since they also modify the extent of the acoustic cavity in which global $p$-modes are trapped, the $p$-mode frequencies are altered. It is these alterations due to structural effects, which are found to contribute to frequency shifts at the $10^{-4}$ level in our models, that our pulsation calculations are intended to model.

In the notation of Gough \& Thompson (1988), in calculating magnetic shifts in $p$-mode frequencies, the present paper includes the contribution from the magnetic distortion to the equilibrium structure of the star, but it does not include the contribution from the perturbed Lorentz force. We justify the neglect of the latter force on the grounds of the small numerical value of $\delta$ in the Sun.

\subsection{Nonmagnetic Models}

We wish to compare the frequencies $p$ (nonmag) obtained from a nonmagnetic model of the Sun with observed frequencies of solar $p$-modes. The observed frequencies, labeled with their (latitudinal) degree $l$ and radial order $n_{r}$ numbers, were obtained from tables that are available online. ${ }^{3}$ The online tables provide lists of frequencies which were extracted from a series of 72 day observing runs of SOHO MDI, starting in 1996. In order to obtain a frequency for any particular mode in the quiet Sun, we averaged over four of the 72 day intervals that are available for the solar minimum year 1996, namely, the MDI sets that are labeled $1216,1288,1360$, and 1432. The average frequency of each mode (in $\mu \mathrm{Hz}$ ) provides the abscissa for Figure 6, ranging from about 1400 to $4000 \mu \mathrm{Hz}$.

A comparison between observed and calculated $(O-C) p$-mode frequencies is demonstrated for some 30 modes in Figure 6 for the two different solar models, M07 and M06, described above ( $\S 3$, opening paragraphs). In Figure 6 we plot results for modes with two values of the harmonic degree $(l=1$ and 10$)$, and with a range of radial orders $\left(n_{r}=6-27\right)$. We see that both solar models show the same pattern for the discrepancies between observed and calculated frequencies. Low-frequency modes are predicted to have frequencies that are smaller than the observed frequencies by several $\mu \mathrm{Hz}$. At high frequencies, the calculated frequencies are larger than the observed frequencies by as much as $10-20 \mu \mathrm{Hz}$. It is likely that the pattern of the $O-C$ differences in Figure 6 is due to deficiencies in the physics of the outermost layers of our solar model: Christensen-Dalsgaard (2003) has demonstrated that by introducing $1 \%-2 \%$ changes in sound speed in the outer $1 \%-2 \%$ of the solar radius, $p$-mode frequencies remain almost unshifted at low frequencies $(\leq 2500 \mu \mathrm{Hz})$, but become shifted by progressively larger amounts at higher frequencies. For modes with frequencies of $4000 \mu \mathrm{Hz}$, the shifts

\footnotetext{
3 See http://quake.stanford.edu/ schou/anavw $72 \mathrm{z}$.
} 


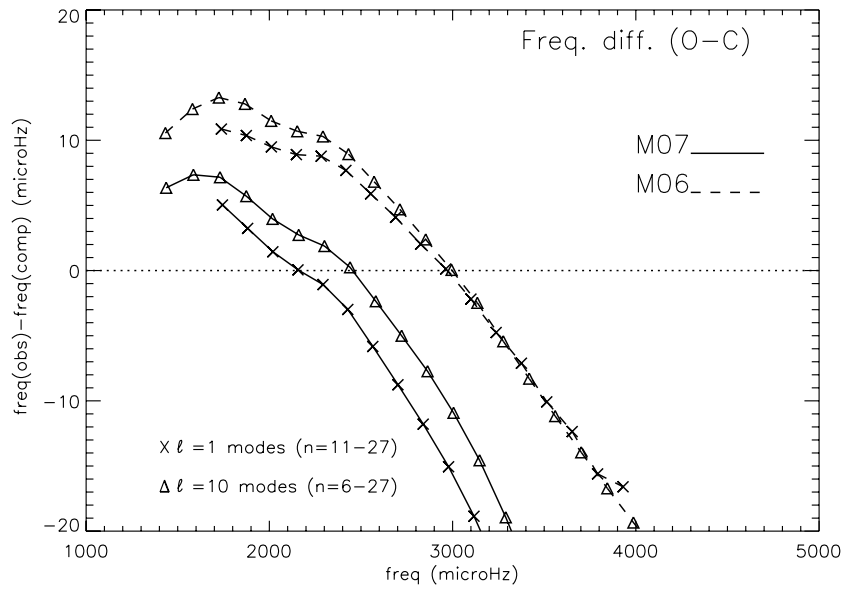

Fig. 6.-Frequency differences $O-C$ in $\mu \mathrm{Hz}$ between observed and computed frequency values for two reference models of the Sun, M06 and M07. Results for modes with $l=1$ and 10 , each with a range of radial orders, are plotted as a function of mode frequency.

are found to be as large as $40 \mu \mathrm{Hz}$, even larger than the $O-C$ discrepancies in Figure 6.

The occurrence of the $O-C$ discrepancies shown in Figure 6 led us to perform extensive numerical experiments with a variety of abundances, opacities, and other physical parameters in order to search for the "best" solar model. Two of the models which emerged from these experiments (M06 and M07) provided the pulsation data in Figure 6. Despite our experiments, we were unable to come up with a model which would reduce the $O-C$ discrepancies to less than a few $\mu \mathrm{Hz}$ over the entire frequency range: the physics of the outermost layers are presumably not being treated adequately in our models. (As a check on the pulsation code, we also inputted the empirical GONG model of the Sun: in that case, the fits were excellent, especially at the lower frequencies, where $O-C$ values of order $1-2 \mu \mathrm{Hz}$ were achieved.)

If our aim had been to obtain precise agreement between $p$-mode frequencies and the observed values in the quiet Sun, then we would have to conclude that our models do not represent significant improvements over other modeling attempts in which, despite inclusion of a range of physical effects, $O-C$ discrepancies of 10-20 $\mu \mathrm{Hz}$ still persist (e.g., Yang et al. 2001; Guzik et al. 2005).

However, we stress that our primary aim in this paper is not to achieve a perfect fit to solar frequencies. Instead, we are interested here in a differential study of nonmagnetic and magnetic models. Specifically, we ask the following question: how much do the frequencies shift between nonmagnetic and magnetic models? Even if the absolute values of the mode frequencies are not as precise as we would like, reliable magnetically induced shifts in frequency can nevertheless be obtained, as we demonstrate in the next subsection.

\subsection{Magnetic Models}

In order to check on the reliability of a differential test, we use magnetic versions of both solar models M06 and M07 as input to the oscillation code and obtain frequencies $f(\mathrm{mag})$ of $p$-modes for the same harmonic degree $l$ and radial order $n_{r}$ as those in Figure 6. Then for each mode, we evaluate the shift in frequency between $f(\mathrm{mag}$ ) and $f$ (nonmag). For modes with $l=10$, and for $\delta_{\text {surf }}=2 \times 10^{-5}$, these shifts are plotted as a function of frequency in Figure 7; the solid and dashed lines refer to the frequency shifts obtained for models M07 and M06, respectively. The crosses indicate the observed frequency shifts of solar modes between

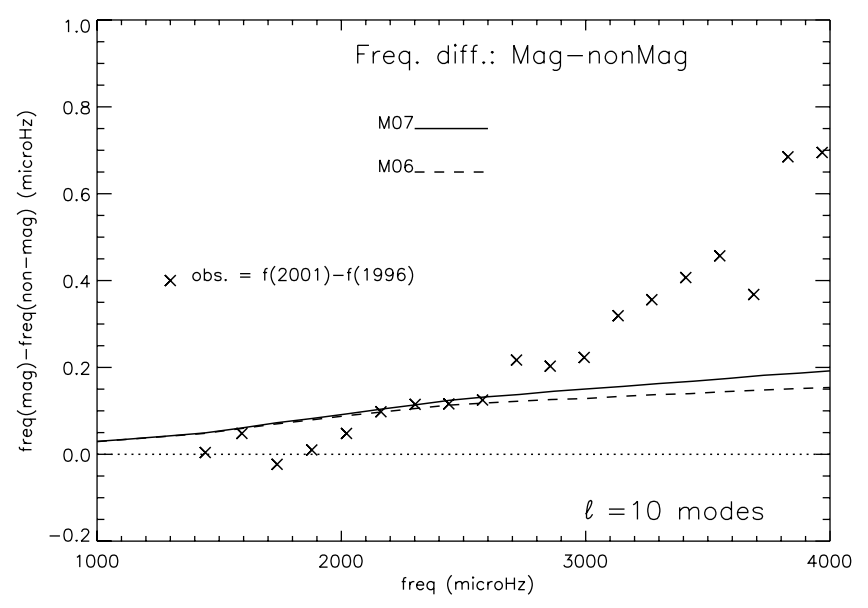

FIG. 7.-Frequency differences between magnetic and nonmagnetic frequency values for two reference models of the Sun, M07 (solid line) and M06 (dashed line). Results for modes with $l=10$ and a range of radial orders are plotted as a function of mode frequency. Crosses denote observed shifts in frequency between solar minimum and solar maximum. Notice that the differences between models M06 and M07 are small compared to the observed shifts.

2001 (solar maximum) and 1996 (solar minimum) in the sense $f(2001)-f(1996)$ : the empirical frequencies were obtained from the tabulated results for observing runs in the year 2001 (see footnote 3) compared to those in 1996 . The essential empirical properties are (1) the frequencies are larger in 2001 than in 1996, and (2) the shifts reach values that are as large as several tenths of a $\mu \mathrm{Hz}$.

We draw attention to certain features of Figure 7.

First of all, and of greatest significance in the present context, is the fact that, even though the absolute frequency of an individual mode differs by as much as $10 \mu \mathrm{Hz}$ between models $\mathrm{M} 06$ and M07 (see Fig. 6), when the magnetic field is applied, the frequency shift for each mode is almost identical for the two models: the solid and dashed lines in Figure 7 do not differ by more than $0.01-0.04 \mu \mathrm{Hz}$. Since the observed shifts in frequency between 1996 (solar minimum) and 2001 (solar maximum) are several tenths of a $\mu \mathrm{Hz}$, we are able to make a meaningful comparison between our model predictions and the data, even if we have not yet identified the best possible model for the nonmagnetic Sun.

Second, the observed frequencies are larger at solar maximum than at solar minimum. As a result, the signs of the empirical frequency shifts as plotted in Figure 7 by crosses are algebraically positive. Our models also find that in the presence of a magnetic field, the $p$-mode frequencies are larger than in the nonmagnetic model. Thus, the sign of our frequency differences agrees with the observed sign. The fact that our models have replicated the correct sign of the frequency shift in the presence of a magnetic field is a nontrivial result: earlier modeling attempts found that, in certain cases, when a field was included, the frequency shifts had the wrong sign. Examples of this can be found in Li et al. (2003): among 18 classes of models that they computed, including everincreasing sophistication of the magnetic modeling, 16 classes yielded frequency shifts of the wrong sign.

Third, our models predict that, for modes with a given $l$, the frequency shifts should increase linearly with increasing frequency, i.e., the lines in Figure 7 should slope upwards to the right. Again, this agrees qualitatively with the observations, although the observed shifts vary more steeply than a linear function.

Although our models have some success in agreeing with the $p$-mode shift data, we have not fitted the data points as well as the model of Goldreich et al. (1991), which includes Lorentz 


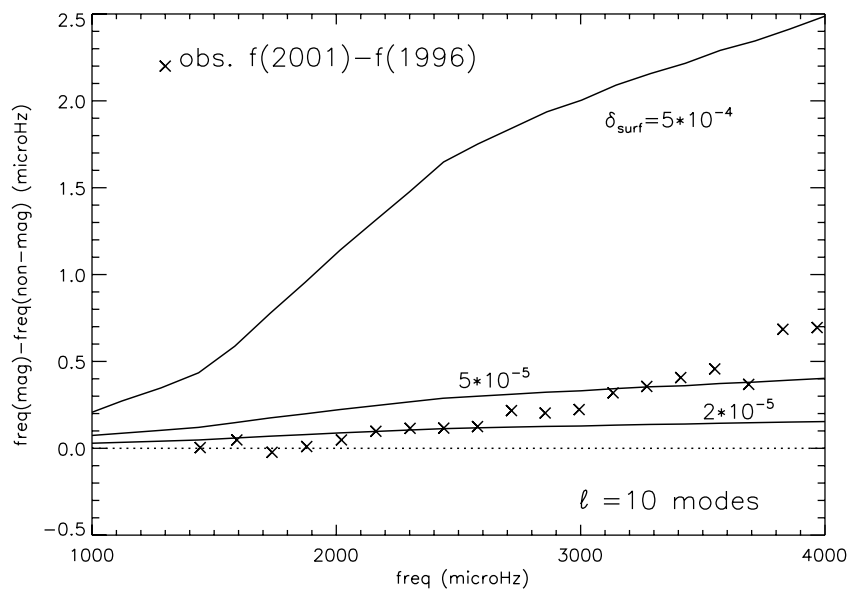

FIG. 8.- Solid curves show frequency shifts of $p$-modes computed for three pairs of models, one containing a magnetic field (with properties described in eqs. [1 [ and [2])), the other being nonmagnetic. For each magnetic field strength (parameterized by the value of $\delta_{\text {surf }}$, see eq. [2]), the frequency shift, in $\mu \mathrm{Hz}$, is plotted against mode frequency for modes with degree $l=10$. The parameter $\delta_{\text {surf }}$ increases with stronger magnetic fields. Crosses denote observed shifts of $l=10$ solar modes between 1996 and 2001.

forces in the pulsation dynamics, or the superadiabatic model of Balmforth et al. (1996). In fact, our results appear more similar to a model reported by Balmforth et al. (1996), in which a thermal disturbance in the solar model is confined to the base of the convection zone. This is consistent with what we mentioned in $\S 2.1$ above: the structural changes in our magnetic models are more significant in the deeper layers of the convection zone.

(Note that in our discussion of magnetic shifts in frequency, we confine attention to frequencies that are less than $4000 \mu \mathrm{Hz}$. Our solar models make no attempt to include a chromosphere, and we are therefore unable to address the behavior of mode shifts at frequencies above $4000 \mu \mathrm{Hz}$ : in order to model the physics of modes at such high frequencies, a chromospheric cavity is required [Goldreich et al. 1991].)

As regards the magnitudes of the frequency shifts in Figure 7, the computed shifts agree best with the observed shifts at frequencies below $2600 \mu \mathrm{Hz}$. At higher frequencies, the observed shifts become larger (by factors of 2 or more) than the model predictions. In order to improve the fit, it is natural to consider a larger value of the magnetic parameter $\delta_{\text {surf }}$. Results are shown in Figure 8 for model M06, again for $l=10$ modes, for two larger field values: $\delta_{\text {surf }}=5 \times 10^{-5}$ and $5 \times 10^{-4}$.

Admittedly, even the lower of these $\delta_{\text {surf }}$ values exceeds the upper limit we estimated in $\S 4.1$ above. But the excess is only a factor of 2: such an excess could be accommodated if the strength of the Sun's poloidal field were 8-17 G, instead of the $6-12 \mathrm{G}$ value used above.

For the same values of $\delta_{\text {surf }}$, we present the computed and observed frequency shifts for $l=1$ modes in Figure 9 (for model M06).

From the results plotted in Figures 8 and 9, it is clear that $\delta_{\text {surf }}=5 \times 10^{-4}$ leads to frequency shifts that are definitely too large (by factors of 5-10) to be consistent with the observed shifts. To the extent that our model is applicable to the "real Sun," this indicates that the global field in the surface layers of the Sun is certainly not as large as $50 \mathrm{G}$.

On the other hand, we see from Figures 8 and 9 that, for the higher frequency modes $(2800-3700 \mu \mathrm{Hz})$, the predictions of the model with $\delta_{\text {surf }}=5 \times 10^{-5}$ fit the data quite well for the

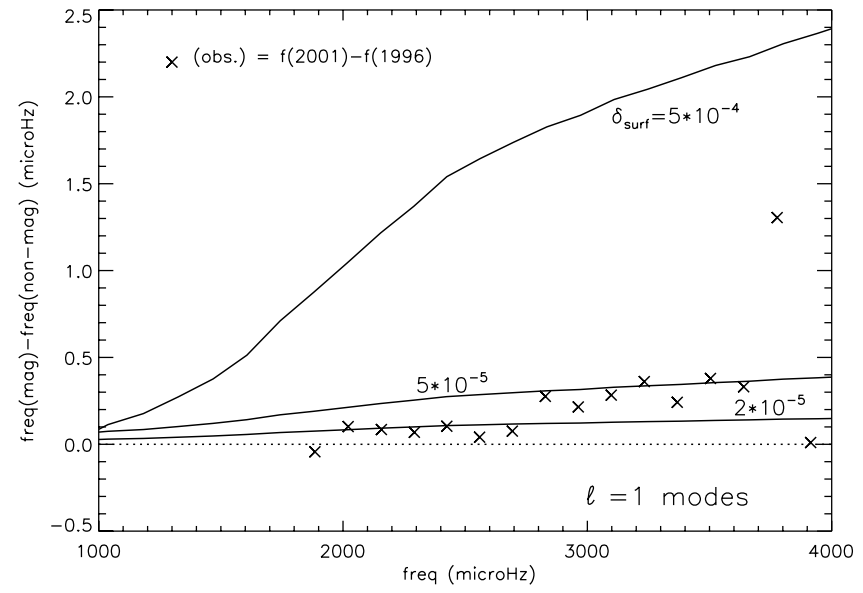

FIG. 9.- As for Fig. 8, except for modes with $l=1$. Crosses denote observed shifts of $l=1$ solar modes between 1996 and 2001 .

$l=1$ modes. This value of $\delta_{\text {surf }}$ also leads to a better fit for the higher frequency $l=10$ modes.

Since the modes with higher frequencies at a given $l$ are modes with larger $n_{r}$ values, such modes are more sensitive to the physical conditions closer to the surface. Perhaps if we were to select a radial profile for $\delta$ that was close to $2 \times 10^{-5}$ deep in the convection zone, and increased to $5 \times 10^{-5}$ in the near-surface material, we could achieve a better fit to the observed shifts. This behavior would require the magnetic field strength in the convection zone to fall off with radius in such a way that $B^{2}$ declines in the outward direction somewhat more slowly than the radial decline in gas pressure. However, we postpone such fine-tuning of the model until we have more confidence in the absolute frequencies of the modes in the nonmagnetic models.

\subsection{Nonhomologous Effects Due to Magnetic Fields}

It is important to understand why the $p$-mode frequencies in our models exhibit an increase in the presence of a magnetic field.

We have already seen ( $\S 4.2$ above) that our models predict that the magnetic Sun should have a smaller radius than the nonmagnetic Sun by a fractional amount $(0.7-3) \times 10^{-5}$. If this "contraction of the cavity" were the only effect at work, the frequencies in the magnetic model would increase relative to the nonmagnetic model by a fractional amount of at most $3 \times 10^{-5}$. This would lead to frequency increases of at most $0.1 \mu \mathrm{Hz}$ for modes at frequencies around $3300 \mu \mathrm{Hz}$. This is too small to be consistent with the observed increases by a few tenths of a $\mu \mathrm{Hz}$.

However, there is another, nonhomologous, effect at work: the radial profile of the sound speed is altered slightly as a result of the structural effects introduced by the magnetic field. As we have stressed above $(\S 2.1)$, these structural effects are largest in the deep convection zone. Specifically, integrating our models from center to surface, we find that the time $T_{s}$ for sound to propagate from center to surface is $3579.862 \mathrm{~s}$ for the nonmagnetic model, and $3579.611 \mathrm{~s}$ for a magnetic model with $\delta_{\text {surf }}=2 \times 10^{-5}$. In our magnetic model, we find that the principal structural change is a slight increase in local temperature in the deep convection zone: this leads to a slightly shorter timescale for sound to propagate from center to surface. The relative reduction in the sound crossing time is almost one part in $10^{4}$.

Now that we have determined numerical values for the sound crossing times, we can derive the asymptotic frequency difference $\Delta \nu$ between adjacent $p$-modes: $\Delta \nu=1 / 2 T_{s}$. This quantity plays 
an important role in determining the frequencies of high-order $p$ modes: modes of radial order $n_{r}$ have frequencies $\nu\left(n_{r}\right) \propto n_{r} \Delta \nu$ (Tassoul 1980). We see that $\Delta \nu$ has the value $139.670 \mu \mathrm{Hz}$ for the nonmagnetic model, and $139.680 \mu \mathrm{Hz}$ for the magnetic model. Of prime importance is the fact that $\Delta \nu$ is larger in the magnetic model: the excess is $0.01 \mu \mathrm{Hz}$. As a result of this difference, the frequency of an $l=1$ mode with radial order $n_{r}$ is expected to be larger in the magnetic model by about $0.01 n_{r} \mu \mathrm{Hz}$ than in the nonmagnetic model. For $n_{r}$ values in the range 20-30 (such as some of those plotted in Figs. 6-9), the magnetic fields would increase the frequencies by $0.2-0.3 \mu \mathrm{Hz}$. Combining these shifts with the shift due to the radius change, we can understand why increases of up to $0.4 \mu \mathrm{Hz}$ are predicted by our models.

Moreover, the proportionality to $n_{r}$ explains the upward slope of the shifts that appear in Figures 7-9.

Note that in the context of Tassoul's formula, the frequency shifts between magnetic and nonmagnetic models are associated with changes in $T_{s}$ : the latter in turn arise from structural changes deep in the convection zone. Changes in luminosity in our model are also associated with the deep structural changes. Thus, changes in luminosity and in $p$-mode frequencies both arise from changes in the deep interior. In contrast, Balmforth et al. (1996) attribute the observed frequency shifts to a "superficial disturbance," but something else is needed to account for the luminosity variation.

\section{DISCUSSION}

\subsection{Amplitudes of Changes in Solar Parameters during the Activity Cycle}

With a single choice of $\delta_{\text {surf }}$, our model reproduces quite well (1) the amplitude of the observed luminosity variations, (2) the amplitudes of the observed $p$-mode frequency shifts, and (3) the upper limit on the observed amplitude of radius variation. Moreover, the value we choose for $\delta_{\text {surf }}$ is not arbitrary: our choice is constrained by empirical data at the surface of the Sun, supplemented by supporting evidence from theoretical considerations beneath the surface.

In contrast, although Goldreich et al. (1991) and Balmforth et al. (1996) succeeded in obtaining good fits to the observed frequency shifts with a certain choice of parameters, those same choices led to luminosity variations that were larger than the observed value by factors of $\geq 10$ and 45 , respectively.

\subsection{Phase Discrepancy in the Luminosity}

We have already noted $(\S 4.4)$ that our models indicate a phase shift of $180^{\circ}$ between magnetic field and luminosity. Empirical data, however, indicate that the solar luminosity is maximum when the sunspot number is maximum: the latter is usually considered to be evidence for maximum field strength. In this sense, our models appear to be subject to a phase discrepancy.

The issue of a phase discrepancy in the luminosity relative to the magnetic fields was discussed by Goldreich et al. (1991). The principal goal of Goldreich et al. (1991) was to use an energy argument to determine how magnetic fields in the Sun would shift the frequencies of radial $p$-modes. By choosing an rms field strength that increased from $190 \mathrm{G}$ at the top of the convection zone to a few $\mathrm{kG}$ at the base of the convection zone, they were able to obtain a good fit to the activity-related frequency shifts. However, their analysis indicated that the increase in magnetic stress between activity minimum and maximum should have led to a $1 \%$ decrease in luminosity, whereas the observations reveal a much smaller $(\leq 0.1 \%)$ increase in the luminosity. Goldreich et al. admitted that the discrepancy in amplitude by a factor of at least 10, as well as the discrepancy in phase, is a "puzzling" result. Although they sug- gested some possibilities to resolve the puzzle, no definitive solutions emerged.

The phase discrepancy was also discussed by Balmforth et al. (1996). These authors found that by altering the mixing length to fit the $p$-mode frequency shifts, there was an accompanying significant change in luminosity. However, "it is in the wrong sense," as well as being 45 times too large an amplitude. Balmforth et al., concluded that the shifts in the $p$-mode frequencies "are not related directly to the luminosity variation."

One possible solution for the phase discrepancy has already been noted in the literature: at solar maximum, not only are sunspots most abundant on the solar surface, but there are also more faculae (Foukal 1990). Enhanced emission from the faculae may more than compensate for the reduction in light output due to spots. This could explain why the Sun's luminosity is largest at sunspot maximum. If this is the correct explanation, then our model cannot address the phase discrepancy: our code deals with a global model of the Sun, and small features such as faculae are beyond the scope of the model.

However, we would like to explore another possible resolution of the phase discrepancy. This has to do with the difference between toroidal and poloidal field components in the Sun. When the Sun is at an activity maximum, and sunspots are most abundant, the toroidal fields are certainly displayed to best advantage. But we recall that, even at "best advantage," sunspots occupy no more than a miniscule fraction of the solar surface area: $95 \%$ of all sunspot groups occupy no more than 0.0003 times the surface area (Bray \& Loughhead 1979). This tiny “filling factor" suggests that the toroidal fields may not deserve to be regarded as the most relevant component in the context of interfering globally with convection: instead, we have argued ( $\S \S 2.3$ and 4.1) that it is the poloidal fields which play a dominant role in the Gough-Tayler parameter $\delta$. In this context, our model suggests that we might look to changes in the poloidal field components as the dominant contributors to global changes in luminosity.

This viewpoint has a significant effect on the phase discrepancy noted above. To see this, we note that the poloidal fields are observed to change sign, i.e., pass through values of zero strength ("polar reversal"), at times which lie close to the epoch when the sunspot counts are at their maximum (e.g., Belov 2000). Therefore, when the toroidal fields are strongest, the poloidal fields are close to being weakest.

In the context of our modeling, the epoch which is usually classified as "solar maximum" (defined as "maximum numbers of sunspots and flares") might be referred to more precisely as a kind of "toroidal field maximum." Close to the very epoch when the toroidal fields are most prominent, the poloidal fields are actually weakest. So what we refer to usually as "solar maximum" might justifiably be referred to as "poloidal (global) field minimum." Our model predicts that when the Sun is undergoing its "global field minimum," the luminosity of the Sun should be maximum. This would be consistent with the empirical result that maximum solar luminosity occurs close to "toroidal field maximum."

Observational evidence that strong toroidal fields may not be the most important physical parameter in controlling certain changes in the Sun during the activity cycle has emerged recently from $p$-mode frequency data. Chaplin et al. (2007) have used a $30 \mathrm{yr}$ data set to analyze the variations in the frequencies of low-degree $p$-modes $(l=0-3)$ over solar cycles 21,22 , and 23 . The goal was specifically to evaluate how well the frequency shifts are correlated with six different proxies of solar activity: (1) the total solar irradiance (TSI), (2) the Kitt Peak Magnetic Index (KPMI), (3) the international sunspot number (ISN), (4) the $10.7 \mathrm{~cm}$ radio flux $\left(F_{10.7}\right),(5)$ the equivalent width of 
the $\mathrm{He}_{\mathrm{I}}$ line at $10830 \AA$, and (6) the ratio of core to wing intensities in the Mg II $h$ and $k$ UV spectral lines. Of these, proxies 1-4 are associated with the strong toroidal fields in active regions. In contrast, proxies 5 and 6 are more sensitive to weakcomponent magnetic flux: e.g., the largest contribution to He I absorption comes from the quiet Sun, and $\mathrm{Mg}$ II core to wing ratio has a large contribution from the chromospheric network. Chaplin et al. found that the highest correlation coefficients between frequency shifts and the various proxies occurred for the weak-field proxies 5 and 6 . For example, in cycle 23, the correlation coefficient between frequency shifts and the weak-field He I proxy was a highly significant 0.956 , whereas the correlation coefficient between frequency shifts and the strong-field TSI was only 0.773 . Chaplin et al. remark that the weak-component magnetic flux "is more widely distributed in latitude than the strong-component flux in the active regions." The work of Chaplin et al. supports our claim that, in the context of activity-related changes in global solar properties, it is worth paying attention to the nonsunspot fields in the Sun. In this context, Goldreich et al. (1991) also pointed out that, as far as the mean square magnetic field in the Sun is concerned, "sunspots are a relatively minor contributor."

We realize that our suggestion of emphasizing the poloidal fields is not without its difficulties. For example, the toroidal field components are not exactly horizontal: therefore, they may contribute a certain amount to the vertical fields which are relevant to the GT criterion. However, in contrast to the global nature of the poloidal fields, the toroidal fields are confined to localized patches: as a result, the vertical components associated with toroidal fields would interfere with convection only in a patchy way. This is in contrast to the poloidal field, which provides vertical fields on an essentially global scale. Another difficulty is that the $p$-mode frequency shifts in our models are found to be positive (as observed at activity maximum) when the Gough-Tayler fields are strongest (see Figs. 7-9): this suggests that activity maximum coincides with maximum Gough-Tayler fields. Therefore, although we would like to ascribe the luminosity phasing to weak Gough-Tayler fields at activity maximum, the phase of the $p$-mode frequency shifts requires strong Gough-Tayler fields at activity maximum. In this regard, it is tempting to recall the suggestion of Balmforth et al. (1996) that the $p$-mode shifts may not be "related directly to the recorded luminosity variations." However, in opposition to this suggestion, we note that our model, with a single choice of global field strength, successfully reproduces the amplitudes of both luminosity variations and $p$-mode shifts. This suggests that the $p$-mode shifts in the Sun are in fact related to the luminosity variations, but the phase discrepancy remains as a problem for our models.

\subsection{Energy Considerations}

With regard to the modeling technique that we use here, Dappen (1983) has pointed to the importance of including magnetic effects in the equation for energy conservation: "Adding terms to the solar structure equations that depend explicitly on time corresponds to adding or subtracting an energy flux to or from the Sun." Changing the internal energy $U_{\text {tot }}$ by a timedependent amount corresponds to a change in luminosity $L=$ $d U_{\text {tot }} / d t$. Our approach here has been to introduce a periodic magnetic field into an otherwise "quietly" evolving star. In its "quiet" evolution, the star derives its energy from nuclear reactions in the core. But the magnetic field, when introduced into the star, also contains a certain amount of energy which has no obvious connection with nuclear reactions.

Dappen's comments prompt the following question: what is the source of this periodic energy? In response to this, and fol- lowing Steiner \& Ferriz-Mas (2005), we suggest that it comes from a dynamo which is driven by differential rotation in the convection zone, or in the tachocline just beneath the convection zone. In this context, periodic increases in magnetic energy would be accompanied by periodic decreases in the rotational energy. Since our modeling does not include treatment of rotational effects, our model cannot claim to give a completely consistent account of all relevant energy sources.

Nevertheless, in view of Dappen's comments, our approach here has been to include magnetic effects not merely in the equation of hydrostatic equilibrium, but also in the equation of energy conservation. However, because the magnetic fields we incorporate are so weak ( $\delta$ of order $10^{-5}$ ), the differences caused by inclusion of magnetic effects in the energy equations turn out to be small in all of the models we report here.

\section{CONCLUSION}

In this paper we have extended a stellar evolution code so as to model cyclic magnetic effects in the Sun. We incorporate two extensions of the customary stellar evolution code: (1) the equations of hydrostatic equilibrium and conservation of energy are modified to include the magnetic pressure and energy density, and (2) the onset of convective instability in the presence of a magnetic field is subject to a criterion that is based on the physics of magnetoconvective instability (Gough \& Tayler 1966). The GT criterion for onset of convection is that the radiative temperature gradient $\nabla_{\text {rad }}$ must exceed the adiabatic gradient $\nabla_{\text {ad }}$ by a finite amount $\delta$. This is a quantitative statement of the physical fact that a (vertical) magnetic field inhibits the onset of convection. For small values of the magnetic inhibition parameter $\delta$, the value of $\delta$ is essentially proportional to the local ratio of magnetic pressure to gas pressure (see eq. [1] above). Given a magnetic field strength at any radial location, there are no free parameters introduced by either items 1 or 2 .

As in all mixing-length theories, the free parameter $\alpha=l_{m} / H_{p}$ enters into our treatment of convection. Once we select an initial heavy-element abundance, $\alpha$ and also the initial helium abundance are determined by the constraints of matching the solar luminosity and radius at the solar age.

The remaining unknown in our model has to do with the choice of the radial profile of $\delta(r)$. In the radiative interior, there is no uncertainty in this regard: the high electrical conductivity requires that $\delta=0$ for fields that vary on timescales as short as $10-100 \mathrm{yr}$. In the convection zone, the surface value of $\delta(r)$ is not a free parameter for the Sun: it is constrained by observations of surface field strength and photospheric pressure. It is only in the choice of the radial profile of $\delta(r)$ within the convection zone that we are confronted with a real unknown. In the present work, we have chosen a single-parameter fit, i.e., a power law in mass: because of the small amount of mass in the convection zone (only $1 \%-2 \%$ of $M_{\odot}$ ), this results in $\delta(r)$ being essentially constant throughout the convection zone. A typical numerical value of $\delta$ in our solar models is $2 \times$ $10^{-5}$. This means that as far as pressure is concerned, our magnetic models differ from the nonmagnetic models by only small amounts, of order 1 part in $10^{5}$.

However, even when $\delta$ is as small as $2 \times 10^{-5}$, the use of the GT criterion leads to other effects which are not small in certain locations. Specifically, our assumption that $\delta$ remains constant as a function of depth in the convection zone has an important implication for structural differences between a nonmagnetic and a magnetic model. In the upper convection zone, where $\nabla_{\text {rad }}$ exceeds $\nabla_{\text {ad }}$ by a large amount, the addition of $\delta=2 \times 10^{-5}$ makes for a hardly noticeable difference between the GT criterion for convective onset $\left(\nabla_{\mathrm{rad}}>\nabla_{\mathrm{ad}}+\delta\right)$ and the (usual) Schwarzschild 
criterion $\left(\nabla_{\text {rad }}>\nabla_{\text {ad }}\right)$. But in the deep convection zone, where $\nabla_{\text {rad }}$ is only slightly larger than $\nabla_{\text {ad }}$, the addition of $\delta$ to $\nabla_{\text {ad }}$ on the right-hand side of the inequality is no longer a small correction. As a result, the use of the GT criterion leads to structural changes which are most significant in the deep convection zone.

The transition to $\delta=0$ in the radiative interior is handled by means of a smooth (Gaussian) curve beneath the base of the convection zone.

We find that in the presence of a field, global properties of the star, including radius and luminosity, undergo systematic changes compared to the nonmagnetic model. Moreover, the frequency of $p$-modes increases in magnetic models compared to a nonmagnetic model of the Sun. The changes in $p$-mode frequencies occur mainly because of nonhomologous changes in solar structure.

In comparison with solar data, when we evaluate $\delta(r)$ on the basis of observed properties of the solar surface layers, we find that our model agrees well with the amplitude of the observed variations in luminosity during the solar cycle. As regards cyclerelated changes in solar radius, our model results are marginally consistent with the upper limit reported by Kuhn et al. (2004) for cycle 23. As regards the shifts in $p$-mode frequencies between solar minimum and solar maximum, our results agree well with the empirical shifts in sign, magnitude, and frequency-dependence at frequencies up to $4000 \mu \mathrm{Hz}$. The fits to the frequency shifts could be improved if the magnetic inhibition parameter $\delta$ were allowed to increase in numerical value by a factor of about 2 between deeper and shallower layers of the convection zone.

Our models predict a $180^{\circ}$ phase shift between magnetic field and luminosity, i.e., the solar luminosity should be maximum when the magnetic field strength which enters into the GoughTayler criterion is at a minimum. At first sight, this seems to be difficult to reconcile with observations, which indicate that the solar luminosity is maximum when sunspot activity is at a maximum (i.e., phase shift apparently equal to zero). Faculae, which cannot be included in our models, may help to explain this phase discrepancy. However, as an alternative explanation for the phase discrepancy, we note the empirical fact that when solar activity is strongest, poloidal fields are weakest. This leads us to wonder whether poloidal fields might be more relevant than toroidal fields as far as the global luminosity of the Sun is concerned.

We thank an anonymous referee for extensive and constructive criticism of an earlier draft of the paper. This research was partially supported by NASA grant NNG04GC75G and by the Delaware Space Grant Program.
Ando, H., \& Osaki, Y. 1975, PASJ, 27, 581

Bahcall, J. N., Serenelli, A. M., \& Basu, S. 2006, ApJS, 165, 400

Balmforth, N. J., Gough, D. O., \& Merryfield, W. J. 1996, MNRAS, 278, 437

Belov, A. 2000, Space Sci Rev., 93, 79

Brandenburg, A., Moss, D., \& Tuominen, I. 1992, A\&A, 265, 328

Bray, R. J., \& Loughhead, R. E. 1979, Sunspots (New York: Dover)

Burgers, J. M. 1969, Flow Equations for Composite Gases (New York: Academic)

Caligari, P., Schuessler, M., \& Moreno-Insertis, F. 1998, ApJ, 502, 481

Chaplin, W. J., Elsworth, Y., Miller, B. A., Verner, G. A., \& New, R. 2007, ApJ, 659,1749

Choudhuri, A. R., \& Gilman, P. A. 1987, ApJ, 316, 788

Christensen-Dalsgaard, J. 2003, Lecture Notes on Stellar Oscillations, (5th ed.; Aarhus: Aarhus Univ.), http://www.phys.au.dk/ jcd/oscilnotes/chap-5.pdf

Dappen, W. 1983, A\&A, 124, 11

Dziembowski, W. A., \& Goode, P. R. 2005, ApJ, 625, 548

Endal, A. S., Sofia, S., \& Twigg, L. W. 1985, ApJ, 290, 748

Foukal, P. V. 1990, Solar Astrophysics (New York: Wiley), 158

Goldreich, P., Murray, N., Willette, G., \& Kumar, P. 1991, ApJ, 370, 752

Gough, D. O. 1990, in Progress of Seismology of the Sun and Stars, ed. Y. Osaki \& H. Shibahashi (Berlin: Springer), 283

Gough, D. O., \& Tayler, R. J. 1966, MNRAS, 133, 85

Gough, D. O., \& Thompson, M. J. 1988, in IAU Symp. 123, Advances in Helio- and Asteroseismology, ed. J. Christensen-Dalsgaard \& S. Frandsen (Dordrecht: Reidel), 155

Gray, D. F., \& Livingston, W. C. 1997, ApJ, 474, 802

\section{REFERENCES}

Guzik, J. A., Watson, L. S., \& Cox, A. N. 2005, ApJ, 627, 1049

Hundhausen, A. J. 1977, in Coronal Holes and High Speed Wind Streams, ed. J. B. Zirker (Boulder: Colorado Assoc. Univ. Press), 299

Iglesias, C. A., \& Rogers, F. J. 1996, ApJ, 464, 943

Kuhn, J. R., Bush, R. I., Emilio, M., \& Scherrer, P. H. 2004, ApJ, 613, 1241

Lawlor, T. M., \& MacDonald, J. 2006, MNRAS, 371, 263

Li, L. H., Basu, S., Sofia, S., Robinson, F. J., Demarque, P., \& Guenther, D. B. 2003, ApJ, 591, 1267

Li, L. H., \& Sofia, S. 2001, ApJ, 549, 1204

Lydon, T. J., \& Sofia, S. 1995, ApJS, 101, 357

MacDonald, J. 1991, ApJS, 76, 369

Mihalas, D. 1978, Stellar Atmospheres (2nd ed.; San Francisco: Freeman), 187

Mullan, D. J. 1974, ApJ, 187, 621

Mullan, D. J., \& MacDonald, J. 2001, ApJ, 559, 353 (MM01)

Paquette, C., Pelletier, C., Fontaine, G., \& Michaud, G. 1986, ApJS, 61, 177

Sofia, S., ed. 1981, Variations of the Solar Constant (NASA Conf. Publ. 2191; Greenbelt: NASA)

Steiner, O., \& Ferriz-Mas, A. 2005, Astron. Nachr., 326, 190 (SFM)

Stothers, R. B. 2006, ApJ, 653, L73

Tassoul, M. 1980, ApJS, 43, 469

Townsend, R. H. D., \& MacDonald, J. 2006, MNRAS, 368, L57

Unno, W., Osaki, Y., Ando, H., Saio, H., \& Shibahashi, H. 1989, Nonradial Oscillations of Stars (2nd ed.; Tokyo: Univ. Tokyo Press)

Yang, J. Y., Li, Y., \& Xu, H. Y. 2001, A\&A, 366, 311 